\title{
Exploring the inner region of type 1 AGNs with the Keck interferometer
}

\author{
M. Kishimoto ${ }^{1}$, S. F. Hönig ${ }^{1}$, R. Antonucci ${ }^{2}$, T. Kotani ${ }^{3}$, R. Barvainis ${ }^{4}$, K. R. W. Tristram ${ }^{1}$, and G. Weigelt ${ }^{1}$ \\ 1 Max-Planck-Institut für Radioastronomie, Auf dem Hügel 69, 53121 Bonn, Germany \\ e-mail: mk@mpifr-bonn.mpg.de \\ 2 Physics Department, University of California, Santa Barbara 93106, USA \\ 3 ISAS, JAXA, 3-1-1 Yoshinodai, Sagamihara, Kanagawa 229-8510, Japan \\ ${ }_{4}$ National Science Foundation, 4301 Wilson Boulevard, Arlington, VA 22230, USA \\ Accepted 20 October 2009 / Accepted 3 November 2009
}

\section{ABSTRACT}

\begin{abstract}
The exploration of extragalactic objects with long-baseline interferometers in the near-infrared has been very limited. Here we report successful observations with the Keck interferometer at $K$-band $(2.2 \mu \mathrm{m})$ for four type 1 AGNs, namely NGC 4151 , Mrk231, NGC 4051, and the QSO IRAS $13349+2438$ at $z=0.108$. For the latter three objects, these are the first long-baseline interferometric measurements in the infrared. We detect high visibilities $\left(V^{2} \sim 0.8-0.9\right)$ for all the four objects including NGC 4151 , for which we confirm the high $V^{2}$ level measured by Swain et al. (2003, ApJ, 596, L163). We marginally detect a decrease of $V^{2}$ with increasing baseline lengths for NGC 4151, although over a very limited range, where the decrease and absolute $V^{2}$ are well fitted with a ring model having a radius of $0.45 \pm 0.04$ mas $(0.039 \pm 0.003 \mathrm{pc})$. Strikingly, this matches independent radius measurements from opticalinfrared reverberations that are thought to be probing the dust sublimation radius. We also show that the effective radius of the other objects, obtained from the same ring model, is either roughly equal to or slightly larger than the reverberation radius as a function of AGN luminosity. This suggests that we are indeed partially resolving the dust sublimation region. The ratio of the effective ring radius to the reverberation radius might also give us an approximate probe for the radial structure of the inner accreting material in each object. This should be scrutinized with further observations.
\end{abstract}

Key words. galaxies: active - galaxies: Seyfert - infrared: galaxies - techniques: interferometric

\section{Introduction}

The exploration of extragalactic objects, or in particular, Active Galactic Nuclei (AGNs), with long-baseline interferometers in the near-infrared (near-IR) has been very limited. While the brightest type 1 AGN NGC 4151 and type 2 AGN NGC 1068 have been observed by Swain et al. (2003) and Wittkowski et al. (2004), respectively, further exploration has been hampered mainly by technical difficulties. Here we report successful observations of four type 1 AGNs with the Keck interferometer (KI) in the near-IR ( $K$-band $2.2 \mu \mathrm{m})$. Type 1 AGNs are thought to give us a direct view of the innermost region of the putative dust torus as well as the central accretion disk, where the interesting effect of the latter should also be carefully evaluated.

\section{Keck interferometry}

\subsection{Observations and data reduction}

We observed four AGNs listed in Table 1 and associated calibrators with the Keck Interferometer (KI, Colavita \& Wizinowich 2003) on 2009 May 15 (UT). These four targets were chosen based on their bright optical magnitudes measured from the preimaging data obtained in April 2009 at Tiki Observatory (French Polynesia) and Silver Spring Observatory (USA) by Teamo, Pelle, and Levin.

The KI combines the two beams from the two Keck $10 \mathrm{~m}$ telescopes which are separated by $85 \mathrm{~m}$ along the direction $38^{\circ}$ east of north. Adaptive Optics correction was implemented at each telescope, locking on the nucleus in the visible wavelengths. The data were obtained with a fringe tracker rate of $200 \mathrm{~Hz}$ operated at $K$-band, while the angle-tracking was performed at $H$-band. The data were first reduced with $\mathrm{Kvis}^{1}$ to produce raw squared visibility $\left(V^{2}\right)$ data averaged over blocks of $5 \mathrm{~s}$ each.

Then those blocks with a phase rms jitter larger than 0.8 radian were excluded. For a given visit of each object, the blocks with an AO wavefront sensor flux smaller than the median by $20 \%$ or more were also excluded. The rejected blocks were $\sim 6 \%$ of all the blocks. They were generally outliers in the visibility measurements for a given visit and quite often were of low fractional fringe-lock time. Then the wide-band side of the data were further reduced using wbCalib ${ }^{2}$ with the correction for the flux ratio between two telescope beams and the correction for a flux bias (slight systematic decrease of the KI's system visibility for lower injected flux ${ }^{3}$ ). The jitter correction was applied with a coefficient of 0.04 (Colavita 1999). Finally the blocks were averaged into scans over a few minutes each, with its error estimated as a standard deviation within a scan.

Figure 1 shows the observed visibilities of all the targets and calibrators (after the corrections above), plotted against the observing time. All the six calibrators used are expected to be unresolved by the KI at $K$-band $\left(V^{2} \geq 0.999\right)$. Overall, the system

\footnotetext{
1 http://nexsci.caltech.edu/software/KISupport/v2/ V2reductionGuide.pdf

2 http://nexsci.caltech.edu/software/V2calib/wbCalib/ index.html

3 http://nexsci.caltech.edu/software/KISupport/ dataMemos/index. shtml
} 


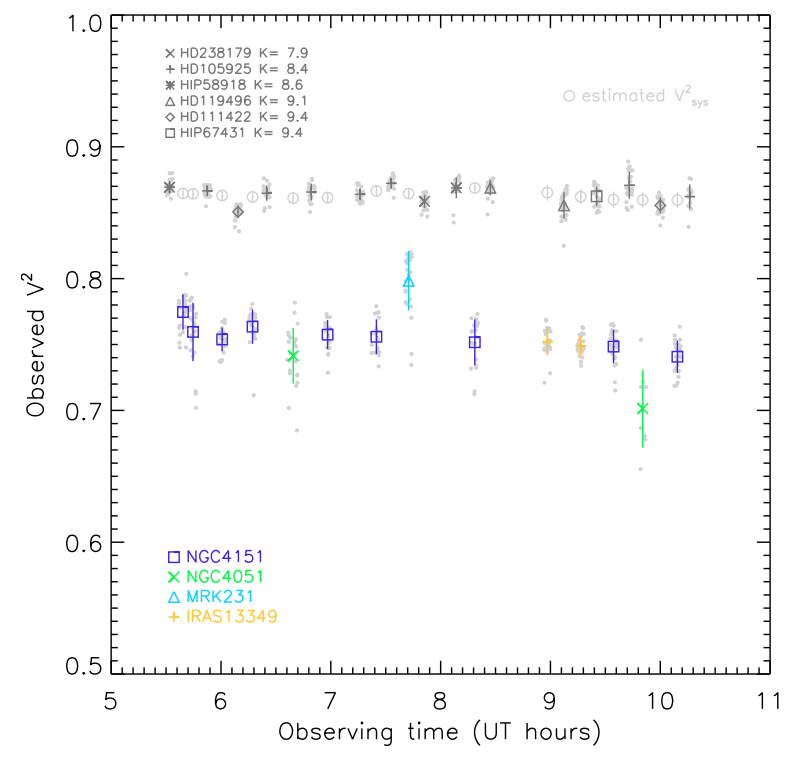

Fig. 1. Observed $V^{2}$ plotted against observing time. Gray dots are individual measurements for blocks of $5 \mathrm{~s}$ each. Gray circles are the estimated system $V^{2}$ at the time of target observations.

visibility ( $V_{\text {sys }}^{2}$ ), as measured by these calibrators, was quite stable over the night. The calibrators span over a relatively wide range of brightness (see the legend in Fig. 1), and one of them (HD 111422) had approximately the same injected flux counts as those of NGC 4151 and Mrk231. Based on the corrected visibilities of these calibrators shown in Fig. 1, the flux bias seems to have been taken out quite well, although there might still be some systematics left. The visibilities of the three faint calibrators $(K>9.1)$ tend to be slightly smaller than those of the other brighter ones, with the difference of the means between the former and latter being $\sim 0.9 \%$ of the means. Therefore we assign 0.01 as a possible systematic uncertainty in system visibility estimations.

Note that for the faintest target NGC 4051, the flux bias correction was effectively used with a slight extrapolation (by $\sim 1$ mag in $K$-band ${ }^{3}$ ). This should be checked with future fainter calibrator observations.

For each target measurement, $V_{\text {sys }}^{2}$ was estimated from these calibrator observations with wbCalib using its time and sky proximity weighting scheme, yielding $V_{\text {sys }}^{2}$ indicated as gray circles in Fig. 1. The final calibrated visibilities are shown in Fig. 2, with the sampled $u v$ points shown in the inset of Fig. 2.

\subsection{Results}

Figure 3 shows the final calibrated visibilities for NGC 4151 as a function of the projected baseline length, enlarged in the left panel of the insets. We confirm the visibility level observed by Swain et al. (2003). The covered range of projected baselines is very limited (note also that the shortest possible for NGC 4151 is $\sim 70 \mathrm{~m}$ due to the KI's delay line restriction). We see, however, a marginal decrease of visibility over the increasing baseline. With the Spearman's rank correlation coefficient analysis, the confidence level is $98.4 \%$, or $2.4 \sigma$. The decrease and absolute level of visibilities are well fitted with a simple thin ring model (i.e. the inner radius equals the outer) having a radius of $0.45 \pm$ 0.04 mas $(0.039 \pm 0.003 \mathrm{pc}$; the error accounts for the systematic uncertainty in $V_{\text {sys }}^{2}$; Sect. 2.1). If we convert each of the visibility measurements into a ring radius and plot it as a function of the

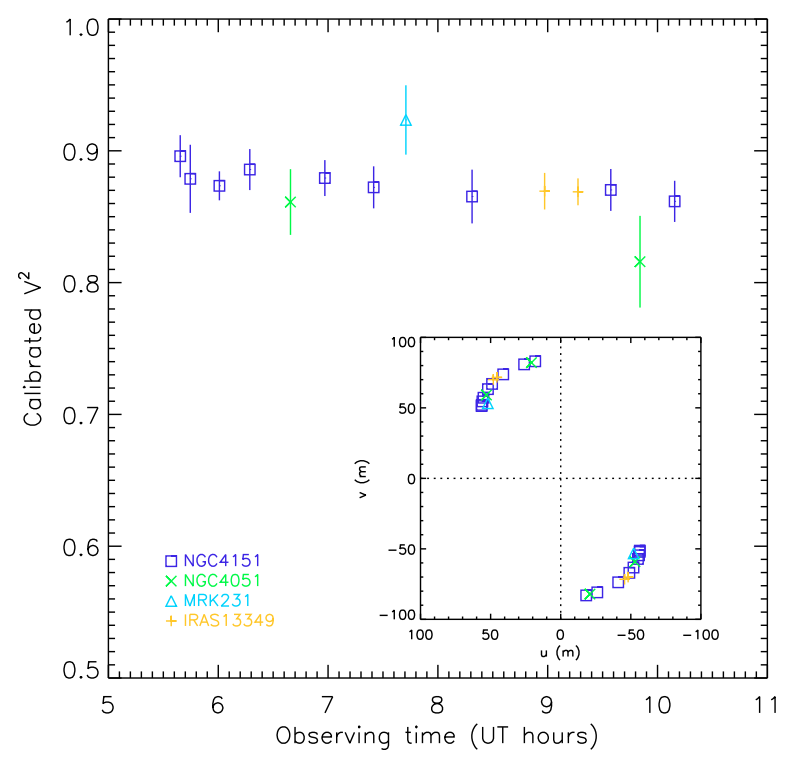

Fig. 2. Calibrated $V^{2}$ plotted against observing time. The inset shows the sampled uv points for each target (north to the top, east to the left).

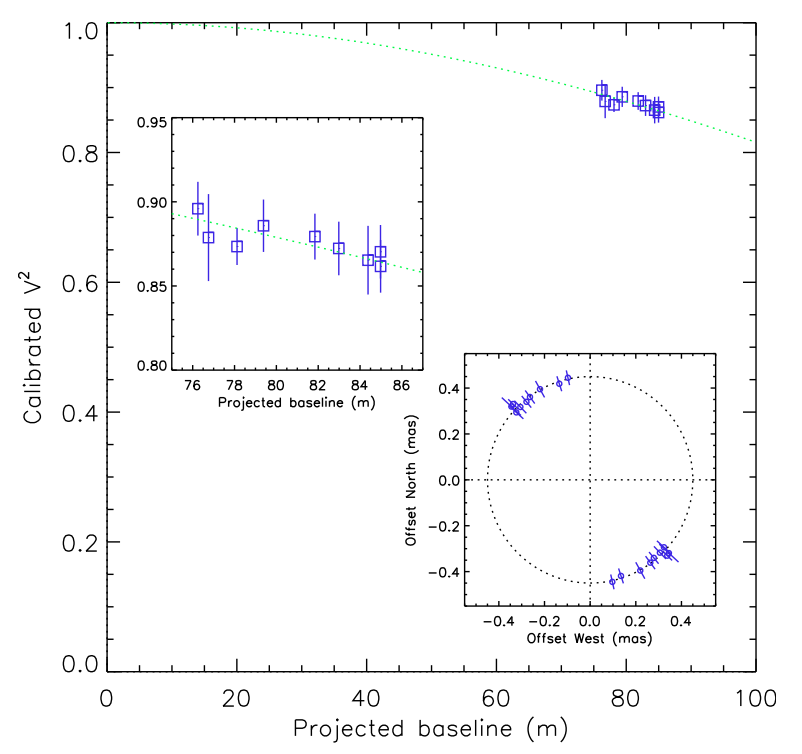

Fig. 3. Calibrated $V^{2}$ for NGC 4151 as a function of projected baselines, enlarged in the left inset. The dotted line shows the best-fit visibility curve of a ring model with a radius of 0.45 mas. In the right inset, ring radii are plotted along the PA of each projected baseline. Note that the correction for the accretion disk and host galaxy contributions is not incorporated in this figure, and it does not change the ring radius significantly (see Table 2).

PA of the projected baseline, we obtain the right panel of the insets in Fig. 3. Over the PA range covered, from $\sim 10^{\circ}$ to $\sim 50^{\circ}$, we do not seem to see a PA dependence of the radius. For all the other targets, the calibrated visibilities are shown in Table 1 together with baseline information and deduced ring radii.

\section{UKIRT imaging and $\mathrm{KI}$ data corrections}

In order to obtain quasi-simultaneous flux measurements for the nuclear point-source in the four type 1 AGNs (which are variable), their images were obtained with WFCAM on UKIRT in five broad-band filters (Table 2) on 2009 June 17 (UT) under the UKIRT service programme. The seeing was $\sim 1$ arcsec. The 
Table 1. Properties of our targets and summary of the results of our KI observations on 15 May 2009 (UT).

\begin{tabular}{|c|c|c|c|c|c|c|c|c|c|c|}
\hline \multirow[t]{2}{*}{ Name } & \multirow{2}{*}{$\begin{array}{l}z^{a} \\
\text { corr. }\end{array}$} & \multirow{2}{*}{$\begin{array}{l}\text { Scale }^{b} \\
\left(\mathrm{pc} \mathrm{mas}^{-1}\right)\end{array}$} & \multirow{2}{*}{$\begin{array}{l}E_{B-V}{ }^{c} \\
(\mathrm{mag})\end{array}$} & \multirow{2}{*}{$\begin{array}{l}B_{p}{ }^{d} \\
(\mathrm{~m})\end{array}$} & \multirow{2}{*}{$\begin{array}{l}\text { PA } \\
\left({ }^{\circ}\right)\end{array}$} & \multirow[t]{2}{*}{$V^{2 e}$} & \multicolumn{2}{|c|}{$R_{\text {ring }} f$} & \multirow{2}{*}{$\begin{array}{l}R_{\tau_{\mathrm{K}}}{ }^{g} \\
(\mathrm{pc})\end{array}$} & \multirow{2}{*}{$\begin{array}{c}A_{V} \\
(\mathrm{mag})\end{array}$} \\
\hline & & & & & & & (mas) & (pc) & & \\
\hline NGC 4151 & 0.00414 & 0.086 & 0.028 & 85.0 & 12.3 & $0.862 \pm 0.018$ & $0.45 \pm 0.04$ & $0.039 \pm 0.003$ & $\sim 0.044 \pm 0.011$ & \\
\hline NGC 4051 & 0.00309 & 0.064 & 0.013 & 79.4 & 41.9 & $0.861 \pm 0.026$ & $0.51 \pm 0.05$ & $0.032 \pm 0.003$ & $\sim 0.011 \pm 0.004$ & \\
\hline MRK231 & 0.0427 & 0.84 & 0.010 & 74.4 & 44.3 & $0.923 \pm 0.028$ & $0.38 \pm 0.07$ & $0.32 \pm 0.06$ & & $1.3^{h}$ \\
\hline IRAS $13349+2438$ & 0.109 & 2.0 & 0.012 & 85.0 & 34.5 & $0.869 \pm 0.016$ & $0.44 \pm 0.03$ & $0.88 \pm 0.05$ & & $0.93^{i}$ \\
\hline
\end{tabular}

${ }^{a} \mathrm{CMB}$ corrected value from NED. ${ }^{b} H_{0}=70 \mathrm{~km} \mathrm{~s}^{-1} \mathrm{Mpc}^{-1}, \Omega_{\mathrm{m}}=0.3$, and $\Omega_{\Lambda}=0.7 .{ }^{c}$ Galactic reddening from Schlegel et al. (1998). ${ }^{d}$ Projected baseline lengths. ${ }^{e}$ For objects with multiple measurements, $V^{2}$ data shown are at the longest baseline length, except for NGC 4051 where the one with a smaller error is shown. ${ }^{f}$ Thin-ring radius (best-fit values if multiple data are available). ${ }^{g}$ Mean and standard deviation of the reverberation measurements from 2001 to 2006 by Koshida et al. (2009) for NGC 4151, and from 2001 to 2003 by Suganuma et al. (2006) for NGC 4051. ${ }^{h}$ Lacy et al. (1982). ${ }^{i}$ Wills et al. (1992).

pipe-line-reduced data were obtained through the WFCAM Science Archive. The wide field of view of the WFCAM gave simultaneous measurements of PSF stars in each AGN field, while a $2 \times 2$ micro stepping gave a good image sampling with an effective pixel size of 0.2 arcsec. We implemented two-dimensional (2D) fits for each image to accurately separate the PSF component from the underlying host galaxy, following the same procedure as described by Kishimoto et al. (2007).

Table 2 lists the measured flux of the nuclear PSF component. The uncertainty of our nuclear PSF flux measurements is estimated as $\sim 5 \%$, based on the residual fluxes after the fits and the flux calibration uncertainty. In the $K$-band images of the two brighter objects, namely NGC 4151 and Mrk231, the central several pixels seemed affected by non-linearity or saturation, so we implemented the $2 \mathrm{D}$ fits by masking the central $\sim 0.4$ arcsec radius region. We estimate that the nuclear PSF flux is recovered within the same uncertainty of 5\%, based on the results from the same masked fits on the other unsaturated images. Using the results of the PSF-host decomposition, we also estimated the host galaxy flux fraction within the field-of-view of the KI, which is $\sim 50$ mas at $K$-band (FWHM; set by a single-mode fiber for the fringe tracker). The results are stated in Table 2. The obtained small values show that the host galaxy contribution is only a very small part of the observed visibility departure from unity.

Figure 4 shows the resulting spectral energy distribution (SED) of the PSF component in each target after the correction for Galactic reddening. We also corrected for the reddening in the host galaxy for the objects which show large Balmer decrements in broad emission lines (Table 2). Assuming that the PSF flux originates from the hot dust thermal emission nearly at the sublimation temperature and from the central accretion disk (AD; thought to be directly seen in type 1 inclinations), we estimate the flux fraction at $K$-band from the latter AD component. Here we fit the SED with a power-law spectrum of the form $f_{v} \propto v^{+1 / 3}$ for the $\mathrm{AD}$, plus a spectrum of a black-body form for the dust (the best-fit temperature was $\sim 1300-1500 \mathrm{~K}$ ). The AD flux fraction at $K$-band is estimated to be as small as $\sim 0.2$ (Table 2), which is in agreement with the results by Kishimoto et al. (2007). This suggests that the high visibilities observed are not due to the unresolved $\mathrm{AD}$, as opposed to the preferred interpretation by Swain et al. (2003) for NGC 4151. The assumed near-IR AD spectral form is based on the recent study of nearIR polarized flux spectra (Kishimoto et al. 2008), but also on various studies on AD spectral shapes in the optical/UV (summarized in Fig. 2 of Kishimoto et al. 2008). By assigning the uncertainty in the AD near-IR spectral index as 0.3 , we also estimated the uncertainty of the $K$-band AD flux fraction (Table 2).

Finally, we corrected the observed visibilities for the host galaxy and the $\mathrm{AD}$ contributions, where the latter is assumed

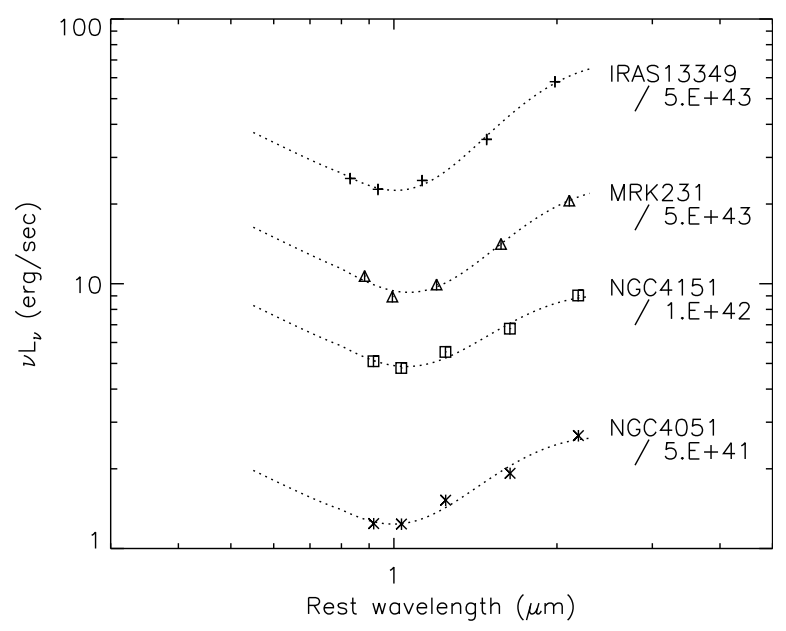

Fig. 4. Flux of the nuclear PSF component in WFCAM images derived from 2D fits. Fitted SEDs are shown in dotted lines (see text).

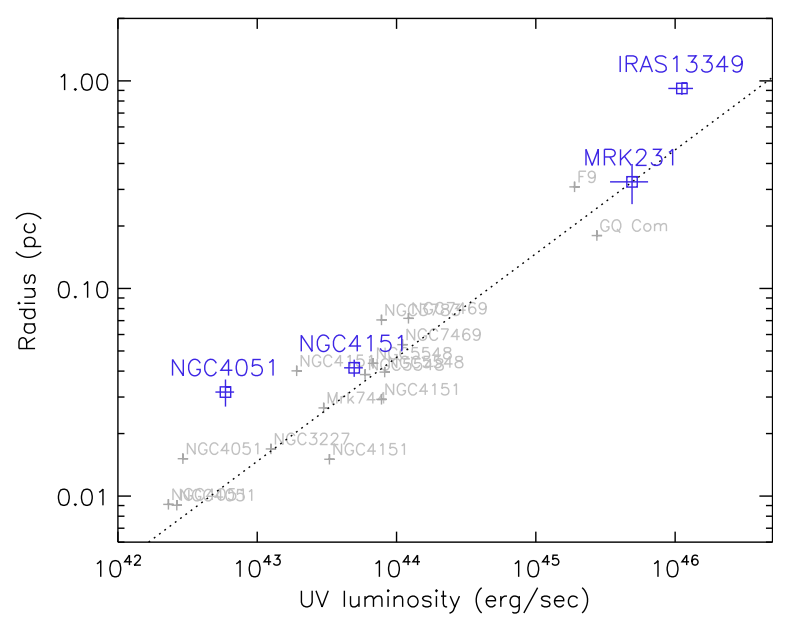

Fig. 5. Corrected ring radius derived for each KI target (squares), plotted against UV luminosity, or a scaled $V$-band luminosity (extrapolated from the WFCAM Z-band flux; see text). Also shown in gray plus signs are the reverberation radii against the same scaled $V$-band luminosity (Suganuma et al. 2006, and references therein) and their fit (dotted line).

to remain unresolved by the KI. The corrected $V^{2}$ as well as corresponding thin ring radii are listed in Table 2.

\section{Interpretations and discussions}

We interpret here the high visibilities observed for all the four objects as an indication of partially resolving the inner brightness distribution of dust thermal emission. 
Table 2. Point-source flux from UKIRT imaging data and the KI results corrected for host galaxy and unresolved AD component.

\begin{tabular}{|c|c|c|c|c|c|c|c|c|c|c|c|c|c|}
\hline \multirow[t]{2}{*}{ Name } & \multicolumn{5}{|c|}{ Flux (mJy) } & \multicolumn{3}{|c|}{ Magnitude } & \multirow{2}{*}{$\begin{array}{c}\operatorname{Host}^{a} \\
(\%)\end{array}$} & \multirow{2}{*}{$\begin{array}{c}\mathrm{AD} \\
\text { fraction }^{b}\end{array}$} & \multirow[t]{2}{*}{$V^{2}$ corrected } & \multirow{2}{*}{$\begin{array}{l}R_{\text {ring }} \text { corr. } \\
\quad(\mathrm{pc})\end{array}$} & \multirow{2}{*}{$\begin{array}{c}R_{\tau_{\mathrm{K}}} \mathrm{fit}^{\mathrm{c}} \\
(\mathrm{pc})\end{array}$} \\
\hline & $Z$ & $Y$ & $J$ & $\bar{H}$ & $\bar{K}$ & $J$ & $H$ & $K$ & & & & & \\
\hline NGC 4151 & 39.7 & 42.4 & 59.4 & 96.3 & 173. & 11.0 & 10.1 & 8.9 & $0.3 \pm 0.1$ & $0.17 \pm 0.06$ & $0.841 \pm 0.024$ & $0.041 \pm 0.004$ & 0.033 \\
\hline NGC 4051 & 8.88 & 9.98 & 14.9 & 24.8 & 46.1 & 12.6 & 11.6 & 10.4 & $1.5 \pm 1.0$ & $0.14 \pm 0.05$ & $0.870 \pm 0.038$ & $0.032 \pm 0.005$ & 0.011 \\
\hline Mrk231 & 21.0 & 22.0 & 33.5 & 71.6 & 152. & 11.7 & 10.4 & 9.1 & $0.5 \pm 0.1$ & $0.15 \pm 0.05$ & $0.921 \pm 0.033$ & $0.33 \pm 0.07$ & 0.33 \\
\hline IRAS 13349 & 7.75 & 8.71 & 12.6 & 26.3 & 62.3 & 12.7 & 11.5 & 10.0 & $0.4 \pm 0.1$ & $0.14 \pm 0.04$ & $0.856 \pm 0.021$ & $0.92 \pm 0.06$ & 0.49 \\
\hline
\end{tabular}

${ }^{a}$ Host galaxy flux fraction at $K$-band estimated for the KI's 50 mas FOV in the AO-corrected images. ${ }^{b}$ AD flux fraction of the point source at $K$-band. ${ }^{c} R_{\tau_{\mathrm{K}}}$ from UV luminosity using the fit by Suganuma et al. (2006). It has an uncertainty of a factor of $\sim 1.5$ based on the scatter of the fit (see text).

As we discussed in Sect. 3, the AD flux fraction at $K$-band is estimated to be small, as long as the assumed power-law AD spectrum in the near-IR, smoothly continuing from the optical, is at least roughly correct. In this case, the $K$-band emission is dominated by the dust emission, and it is reasonable to convert the observed visibility to a thin ring radius to obtain an approximate effective radius of the dust brightness distribution for each object. (We have corrected $V^{2}$ for the unresolved AD contribution, but the correction is quite small; Table 2.) The derived ring radii are plotted in parsec in Fig. 5 against the UV luminosity $L$, here defined as a scaled $V$-band luminosity of $6 v f_{v}(V)$ (Kishimoto et al. 2007). The $V$-band flux is extrapolated from the fitted flux at $0.8 \mu \mathrm{m}$ (Fig. 4) assuming an AD spectral shape of $f_{v} \propto v^{0}$ (based on spectral index studies referred to above).

We can directly compare these ring radii with another type of independent radius measurements $R_{\tau_{\mathrm{K}}}$, namely the light traveling distance for the time lag of the $K$-band flux variation from the UV/optical variation (Suganuma et al. 2006). These reverberation radii are also plotted against the same scaled $V$-band luminosity in Fig. 5. They are known to be approximately proportional to $L^{1 / 2}$ (Suganuma et al. 2006; the dotted line in Fig. 5 shows their fit), and are likely to be probing the dust sublimation radius in each object. We first see that $R_{\text {ring }}$ is roughly comparable to $R_{\tau_{\mathrm{K}}}$ for all the four objects, and thus $R_{\text {ring }}$ is roughly scaling also with $L^{1 / 2}$. This approximate match suggests that the KI data are indeed partially resolving the dust sublimation region.

With a closer look at Fig. 5, we see that $R_{\text {ring }}$ is either roughly equal to or slightly larger than $R_{\tau_{\mathrm{K}}}$ (i.e. $R_{\text {ring }} / R_{\tau_{\mathrm{K}}} \gtrsim 1$, up to a factor of a few), though we have only four objects. This could be understood if $R_{\tau_{\mathrm{K}}}$ is tracing a radius close to the innermost boundary radius of the dust distribution. It is known that the cross-correlation lag tends to trace an inner radius of the responding particles' distribution when the lag is determined from the peak in the cross-correlation function (e.g. Koratkar \& Gaskell 1991, and references therein), as is the case for the data used in the fit by Suganuma et al. On the other hand, $R_{\text {ring }}$ is an effective, average radius over the radial dust brightness distribution in the $K$-band. When the radial distribution is very steep and compact, the ratio $R_{\text {ring }} / R_{\tau_{\mathrm{K}}}$ would become very close to unity (such as seen in NGC 4151 and Mrk231), while for a flatter, more extended distribution, $R_{\text {ring }} / R_{\tau_{\mathrm{K}}}$ would show a larger departure from unity.

If our interpretation above is correct, the KI data would conversely support the dust sublimation radius as probed by the reverberation measurements that is smaller by a factor of about three than that inferred for typical ISM-size Graphite grains (Barvainis 1987, $0.05 \mu \mathrm{m}$ radius) for a given $L$ (Kishimoto et al. 2007). The small sublimation radius could be due to the possible dominance of large grains in the innermost region, since they can sustain at a much closer distance to the illuminating source for a given sublimation temperature. Alternatively, it could be due to an anisotropy of the AD radiation (see Kishimoto et al. 2007, for more details).

In Fig. 5, the reverberation and ring radii are shown essentially as a function of an instantaneous $L$ at the time of each corresponding radius measurement. Koshida et al. (2009) have however recently shown that $R_{\tau_{\mathrm{K}}}$ is not exactly scaling with the instantaneous $L$ varying in a given object. It could be that $R_{\tau_{\mathrm{K}}}$ tends to give a dust sublimation radius that corresponds to a relatively long-term average of $L$. On the other hand, $R_{\tau_{\mathrm{K}}}$ does show the $L^{1 / 2}$ proportionality over a sample of objects. Thus, when we compare $R_{\tau_{\mathrm{K}}}$ and $R_{\text {ring }}$, unless simultaneous measurements exist, we would have to allow for the uncertainty in $R_{\tau_{\mathrm{K}}}$, as a function of instantaneous $L$, being the scatter in the $L^{1 / 2}$ fit $(\sim 0.17 \mathrm{dex})$.

If the $\mathrm{AD}$ spectrum does not have a power-law shape but rather has some red turn-over in the near-IR, though the nearIR polarized flux spectrum argues against this (Kishimoto et al. 2008), the AD flux fraction at $K$-band would become higher than we estimated here. However, even if the flux fraction is as large as 0.5 , the corrected ring radius would become larger than stated in Table 2 by a factor of only $\sim 1.3$, resulting in no qualitative change in our discussion. Future near-IR interferometry with much longer baselines can conclusively confirm that the visibility is decreasing as we inferred from the present KI data. We plan to advance our exploration with further interferometric measurements in the infrared.

Acknowledgements. The data presented herein were obtained at the W.M. Keck Observatory, which is operated as a scientific partnership among the California Institute of Technology, the University of California and the National Aeronautics and Space Administration. The Observatory was made possible by the generous financial support of the W.M. Keck Foundation. We are grateful to all the staff members whose huge efforts made these Keck interferometer observations possible. The United Kingdom Infrared Telescope is operated by the Joint Astronomy Centre on behalf of the Science and Technology Facilities Council of the U.K. We thank N. Teamo, J. C. Pelle and K. Levin for kindly providing the pre-imaging data, and F. Millour for helpful discussions. This work has made use of services produced by the NASA Exoplanet Science Institute at the California Institute of Technology.

\section{References}

Barvainis, R. 1987, ApJ, 320, 537

Colavita, M. M. 1999, PASP, 111, 111

Colavita, M. M., \& Wizinowich, P. L. 2003, in SPIE Conf. Ser. 4838, ed. W. A. Traub, 79

Kishimoto, M., Hönig, S. F., Beckert, T., \& Weigelt, G. 2007, A\&A, 476, 713

Kishimoto, M., Antonucci, R., Blaes, O., et al. 2008, Nature, 454, 492

Koratkar, A. P., \& Gaskell, C. M. 1991, ApJS, 75, 719

Koshida, S., Yoshii, Y., Kobayashi, Y., et al. 2009, ApJ, 700, L109

Lacy, J. H., Malkan, M., Becklin, E. E., et al. 1982, ApJ, 256, 75

Schlegel, D. J., Finkbeiner, D. P., \& Davis, M. 1998, ApJ, 500, 525

Suganuma, M., Yoshii, Y., Kobayashi, Y., et al. 2006, ApJ, 639, 46

Swain, M., Vasisht, G., Akeson, R., et al. 2003, ApJ, 596, L163

Wills, B. J., Wills, D., Evans, II, N. J., et al. 1992, ApJ, 400, 96

Wittkowski, M., Kervella, P., Arsenault, R., et al. 2004, A\&A, 418, L39 